\title{
GRAM LINES AND THE AVERAGE OF THE REAL PART OF THE RIEMANN ZETA FUNCTION
}

\author{
KEVIN A. BROUGHAN AND A. ROSS BARNETT
}

\begin{abstract}
The contours $\Im \Lambda(s)=0$ of the function which satisfies $\zeta(1-s)=\Lambda(s) \zeta(s)$ cross the critical strip on lines which are almost horizontal and straight, and which cut the critical line alternately at Gram points and points where $\zeta(s)$ is imaginary. When suitably averaged the real part of $\zeta(s)$ satisfies a relation which greatly extends a theorem of Titchmarsh, (namely that the average of $\zeta(s)$ over the Gram points has the value 2), to the open right-hand half plane $\sigma>0$.
\end{abstract}

\section{INTRODUCTION}

First we set out some standard notation and properties. Let $s=\sigma+i t$ and let $\Phi(s)=\frac{1}{2} s(s-1) \pi^{-s / 2} \Gamma(s / 2)$, so $\xi(s)=\Phi(s) \zeta(s)$ satisfies $\xi\left(\frac{1}{2}+s\right)=\xi\left(\frac{1}{2}-s\right)$, a form of the functional equation, and also $\xi\left(\frac{1}{2}+i t\right)$ is real for $t \in \mathbb{R}$.

Write

$$
\xi\left(\frac{1}{2}+i t\right)=-f(t) e^{i \vartheta(t)} \zeta\left(\frac{1}{2}+i t\right),
$$

where $f(t)$ is real and positive, and then define $Z(t)=e^{i \vartheta(t)} \zeta\left(\frac{1}{2}+i t\right)$, where as usual we define

$$
\vartheta(t)=\Im \log \Gamma\left(\frac{1}{4}+\frac{i t}{2}\right)-\frac{t \log \pi}{2},
$$

so that $Z(t)$ is real when $t$ is real and its zeros in $\mathbb{R}$ correspond to the zeros of $\zeta(s)$ on $\sigma=\Re s=\frac{1}{2}$, the critical line.

Define Gram points $\left\{\frac{1}{2}+i g_{n}\right\}_{n \geq-1}$ as points on the critical line which satisfy $\vartheta\left(g_{n}\right)=n \pi$. Then at these points $\zeta(s)$ is real. Gram points are well known and have been used extensively in the past to count zeros of $Z(t)$. See, for example, [5, 6, 12. Now let $\Lambda(s)=2(2 \pi)^{-s} \Gamma(s) \cos \left(\frac{\pi s}{2}\right)$ so $\zeta(1-s)=\Lambda(s) \zeta(s)$ - another form of the functional equation. By applying the equation $\Gamma(s) \Gamma(1-s)=\pi / \sin (\pi s)$ it follows that $\Lambda(s) \Lambda(1-s)=1$ and hence that $\Lambda(s)=\Phi(1-s) / \Phi(s)$.

Now we summarize the content of this paper regarding Gram lines (defined below) and average values of the real part of $\zeta(s)$ on vertical lines through them. The origin of the ideas underlying these results was the observation of the phase portrait of $\dot{s}=\Lambda(s)$. See Figure 1 and the article [3] for background material on this approach.

The contours $\Im \Lambda(s)=0$ of the function cross the critical strip on lines which are almost horizontal and straight and cut the critical line at (1) the Gram points

Received by the editor November 28, 2010 and, in revised form, March 25, 2011 and April 15, 2011.

2010 Mathematics Subject Classification. Primary 11M06.

Key words and phrases. Gram points, Gram lines, Riemann zeta function.

(C)2011 American Mathematical Society 
(so they are called simply "Gram lines") or (2) the points where $\zeta(s)$ is imaginary. The contours are shown to satisfy the equations, for $n \in \mathbb{Z}$,

$$
\frac{t}{2} \log \frac{t}{2 \pi}-\frac{t}{2}-\frac{\pi}{8}+\frac{1}{48 t}-\frac{\left(\sigma-\frac{1}{2}\right)^{2}}{4 t}+O\left(\frac{1}{t^{3}}\right)=\frac{n \pi}{2} .
$$

They differ from horizontal lines by $O(1 / t)$. On these contours $\Re \Lambda(s)$ is never zero and is strictly monotonic with

$$
\Re \Lambda(s)=(-1)^{n}\left(\frac{t}{2 \pi}\right)^{\sigma-\frac{1}{2}}\left\{1+O\left(\frac{1}{t^{2}}\right)\right\} .
$$

This monotonicity is then applied to zeta to show that the modulus of the derivatives, at simple zeros symmetric with respect to the critical line, is never the same.

It is then shown that the contours $\Im \Phi(s)=0$ also cut the critical line at the Gram points but at an angle to the horizontal which is asymptotically $-\pi /(2 \log t)$. They differ from intervals at that slope by $O(1 / t)$ and have equations given implicitly by

$$
\frac{t}{2} \log \frac{t}{2 \pi}-\frac{t}{2}+\frac{\pi(\sigma-1)}{4}+\frac{1}{12 t}-\frac{(\sigma-1)^{2}}{4 t}+O\left(\frac{1}{t^{3}}\right)=n \pi .
$$

The main result of the paper is then given as a corollary to Theorem 1. A theorem of Titchmarsh [10, gives 2 as the average of the real part of $\zeta\left(\frac{1}{2}+i g_{n}\right)$ i.e.,

$$
\lim _{N \rightarrow \infty} \frac{1}{N} \sum_{1 \leq n \leq N}\left[\Re \zeta\left(\frac{1}{2}+i g_{n}\right)-2\right]=0 .
$$

When, for fixed values of $\sigma$, the real part of $\zeta\left(\sigma+i g_{n}\right)$ is modified and then averaged over its values, it satisfies a relation which extends this theorem.

Theorem 1. Let $N$ be a positive integer and let $\sigma>0$ be a given positive real number. Then as $N \rightarrow \infty$ :

$$
\begin{aligned}
\sum_{1 \leq n \leq N} & {\left[\Re \zeta\left(\sigma+i g_{n}\right)-1-\left(\frac{g_{n}}{2 \pi}\right)^{\frac{1}{2}-\sigma}\right] } \\
= & O\left(N^{\max \left\{1-\frac{\sigma}{2}, \frac{1}{2}\right\}}(\log N)^{\max \left\{\frac{1}{2}+\frac{\sigma}{2}, 2\right\}}\right),
\end{aligned}
$$

uniformly in $\sigma>0$.

It follows directly that if $0<\sigma$, then

$$
\lim _{N \rightarrow \infty} \frac{1}{N} \sum_{1 \leq n \leq N}\left[\Re \zeta\left(\sigma+i g_{n}\right)-1-\left(\frac{g_{n}}{2 \pi}\right)^{\frac{1}{2}-\sigma}\right]=0 .
$$

The proof of Theorem 1 shows that the values of $s$ in $\zeta(s)$ used for these averages may be chosen either on the Gram line through $g_{n}$ or on the corresponding horizontal interval. We have chosen the latter for simplicity. Also Titchmarsh starts his summations at a variable $M$ rather than 1, but the two forms are equivalent when $M$ is fixed.

The numerical evidence for this theorem is very strong, with better convergence than that obtained analytically in Theorem 1. See Figure 3. Also, this numerical evidence indicates that the average result should apply beyond the critical strip to the left, but we have been unable to prove this. 


\section{Motivation FOR THIS STUDY}

Let $T$ and e be positive real numbers and let $R:=[-\mathbf{e}, 1+\mathbf{e}] \times[0, T]$ be a rectangle. Let $N(T)$ be the number of zeros of $\zeta(s)$ inside $R$, counted with multiplicity. Then [4, page 128], if $C$ is the interval from $1+\mathbf{e}+i T$ to $\frac{1}{2}+i T$ on the boundary of $R$ and it happens that $\Re \zeta(s)>0$ on all of $C$, then the complex $\operatorname{logarithm} \log \zeta(s)$ is uniquely defined along $C$ and there

$$
(\log \zeta(s))^{\prime}=\frac{\zeta^{\prime}(s)}{\zeta(s)}
$$

Since, via the principle of the argument,

$$
N(T)=\frac{\vartheta(T)}{\pi}+1+\frac{1}{\pi} \Im \int_{C} \frac{\zeta^{\prime}(s)}{\zeta(s)} d s
$$

then, with the given positive property of $\Re \zeta(s)$ on $C$, this last integral has a value in $\left(-\frac{1}{2}, \frac{1}{2}\right)$, so $N(T)$ is exactly the nearest integer to $\frac{\vartheta(T)}{\pi}+1$.

Now, it is a matter of observation that the real part of $\zeta(s)$ has a strong tendency to be positive on Gram lines. Although we have not been able to find a lower bound for the proportion of lines on which this occurs, Theorem 1 and its corollaries provide strong evidence that it is so. Hence the object of this study: to take steps towards proving that, if $\|x\|$ represents the closest integer to any real $x$, then $N\left(g_{n}\right)=\left\|\frac{\vartheta\left(g_{n}\right)}{\pi}+1\right\|$ for an infinite number of positive integers $n$.

\section{Contours Where $\Lambda(s)$ AND $\Phi(s)$ ARE REAL}

In this section we consider where the multiplier functions which appear in forms of the functional equation are real. Expressions for these subsets of the complex plane are developed in a number of lemmas. Figure 2 shows a few contours where $\Im \Lambda(s)=0$.

Lemma 2. (a) The contours $\Im \Lambda(s)=0$ cut across the critical strip symmetrically.

(b) They cut the critical line at the points $s=\frac{1}{2}+i \gamma$ where $\zeta(s)$ is real (Gram points) or imaginary, with value $\Lambda(s)= \pm 1$. If $\Lambda(s)=1$, then $\zeta(s)$ is real and if $\zeta(s)$ is a simple zero it is a center. If $\Lambda(s)=-1$, then $\zeta(s)$ is imaginary and if $\zeta(s)$ is a simple zero it is a node.

(c) If $\Im(s) \neq 0$ then $\Lambda(s) \neq 0$.

Proof. (a) For real $x, t>0$, let $P_{+}=\frac{1}{2}+x+i t$ and $P_{-}=\frac{1}{2}-x+i t$. Because $\Lambda\left(P_{+}\right) \Lambda\left(\overline{P_{-}}\right)=1$, if $\Lambda\left(P_{+}\right)$is real, so is $\Lambda\left(P_{-}\right)$. Therefore the contours are symmetric about $\sigma=\frac{1}{2}$.

(b) If $s=\frac{1}{2}+i t$ and $\Im \Lambda(s)=0$, then $\Lambda(s)$ and $\Lambda(\bar{s})$ are real. Therefore $\Lambda\left(\frac{1}{2}+i t\right) \Lambda\left(\frac{1}{2}-i t\right)=1$ implies $\Lambda\left(\frac{1}{2}+i t\right)^{2}=1$, so $\Lambda\left(\frac{1}{2}+i t\right)= \pm 1$. If $\Lambda\left(\frac{1}{2}+i t\right)=1$, by the functional equation $\zeta(1-s)=\Lambda(s) \zeta(s), \overline{\zeta\left(\frac{1}{2}+i t\right)}=\zeta\left(\frac{1}{2}-i t\right)=\zeta\left(\frac{1}{2}+i t\right)$ so $\zeta\left(\frac{1}{2}+i t\right)$ is real. If $\Lambda\left(\frac{1}{2}+i t\right)=-1$, then $\overline{\zeta\left(\frac{1}{2}+i t\right)}+\zeta\left(\frac{1}{2}+i t\right)=\zeta\left(\frac{1}{2}-i t\right)+\zeta\left(\frac{1}{2}+i t\right)=$ $-\zeta\left(\frac{1}{2}+i t\right)+\zeta\left(\frac{1}{2}+i t\right)=0$, so $\zeta\left(\frac{1}{2}+i t\right)$ is pure imaginary.

If $P=\frac{1}{2}+i t$ is a simple zero of $\zeta(s)$ and $\Lambda(P)=1$, then differentiating the functional equation gives $-\zeta^{\prime}(\bar{P})=\zeta^{\prime}(P)$, so $\zeta^{\prime}(P)$ is imaginary and the zero is a center. If $\Lambda(P)=-1, \zeta^{\prime}(P)$ would be real, so the zero is a node.

(c) Because $\Gamma(s)$ is never zero and the zeros of $\cos (\pi s / 2)$ are on the real axis, if $\Im(s) \neq 0$, then $\Lambda(s) \neq 0$. 


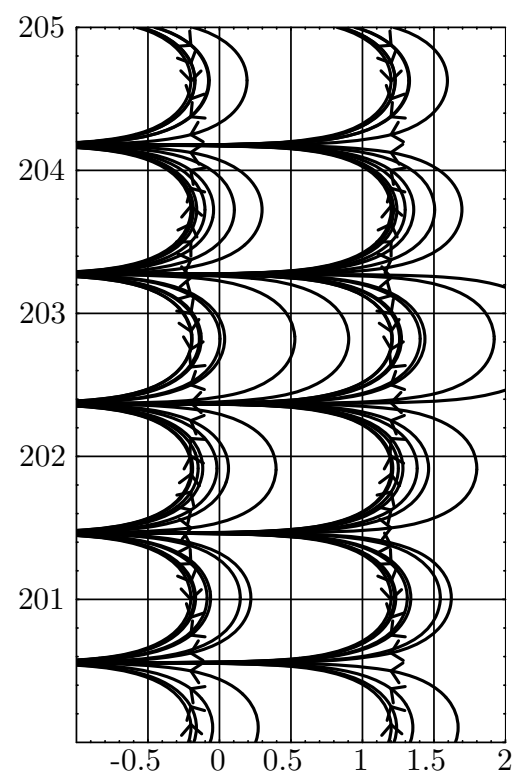

Figure 1. Phase portrait for $\dot{s}=\Lambda(s)$ in the rectangle $-1 \leq \sigma \leq 2$ and $200 \leq t \leq 205$.

Lemma 3. The contours of $\Im \Lambda(s)=0$ differ from intervals parallel to the real axis through those points by $O(1 / t)$. Indeed, the contours satisfy the equations, for $n \in \mathbb{Z}$,

$$
\frac{t}{2} \log \frac{t}{2 \pi}-\frac{t}{2}-\frac{\pi}{8}+\frac{1}{48 t}-\frac{\left(\sigma-\frac{1}{2}\right)^{2}}{4 t}+O\left(\frac{1}{t^{3}}\right)=\frac{n \pi}{2},
$$

where $n$ is even for lines through Gram points and $n$ is odd for lines through the points where $\zeta(s)$ is imaginary.

Proof. (Because the manipulation is somewhat lengthy, but the steps easy to describe, we simply describe those steps taken in obtaining the given equation.) Expand the equation $\Lambda(s)=\Lambda(\bar{s})$ using the asymptotic Stirling series approximation for the gamma function

$$
\log \Gamma(s)=\left(s-\frac{1}{2}\right) \log s-s+\frac{1}{2} \log 2 \pi+\frac{1}{12 s}+O\left(\frac{1}{t^{3}}\right),
$$

retaining terms of order $1 / t$. The $O\left(1 / t^{3}\right)$ error follows by implicit differentiation and the mean value theorem, because the term of order $1 / t$ is continuous.

Note that

$$
\vartheta(t)=\frac{t}{2} \log \frac{t}{2 \pi}-\frac{t}{2}-\frac{\pi}{8}+\frac{1}{48 t}+\frac{7}{5760 t^{3}}+O\left(\frac{1}{t^{5}}\right)
$$

satisfies $Z(t)=e^{i \vartheta(t)} \zeta\left(\frac{1}{2}+i t\right)$ which is real when $t$ is real. Note also that the expression in the lemma above gives the correct value for Gram points at $\sigma=\frac{1}{2}$. 


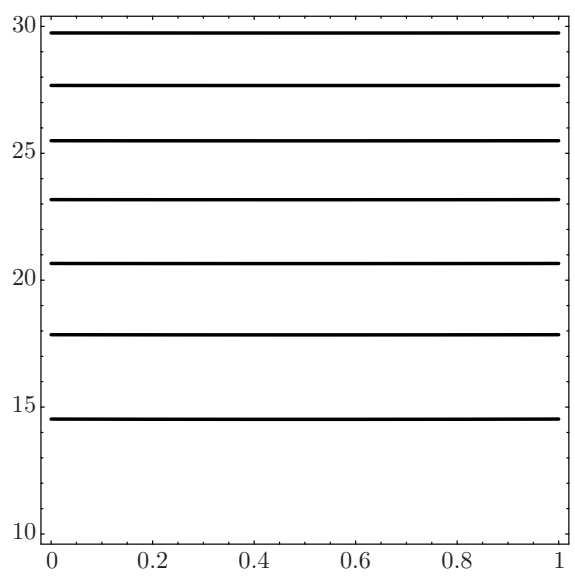

Figure 2. Some contours for $\Im \Lambda(s)=0$ in the rectangle $0 \leq t \leq$ 30 and $0 \leq \sigma \leq 1$.

Lemma 4. For $t$ sufficiently large, $\Re \Lambda(s)$ is strictly monotonic on the contours $\Im \Lambda(s)=0$, with

$$
\Re \Lambda(s)=(-1)^{n}\left(\frac{t}{2 \pi}\right)^{\sigma-\frac{1}{2}}\left(1+O\left(\frac{1}{t^{2}}\right)\right) .
$$

Proof. Consider the equation $2 \Re \Lambda(s)=\Lambda(s)+\Lambda(\bar{s})$ where $t>0$ is sufficiently large. Expand the trigonometric terms as exponentials and set those with $-t$ in the exponent to zero. Then use the Stirling series approximation for $\log \Gamma(s)$ to obtain the expression

$$
\begin{aligned}
\Re \Lambda(s)= & \exp \left(\left(\sigma-\frac{1}{2}\right) \log \left(\frac{t}{2 \pi}\right)+O\left(\frac{1}{t^{2}}\right)\right) \\
& \times \cos \left(2\left(\frac{t}{2} \log \frac{t}{2 \pi}-\frac{t}{2}-\frac{\pi}{8}+\frac{1}{48 t}-\frac{\left(\sigma-\frac{1}{2}\right)^{2}}{4 t}+O\left(\frac{1}{t^{3}}\right)\right)\right) \\
= & \exp (f(\sigma, t)) \times \cos \left(2 \vartheta_{\sigma}(t)\right),
\end{aligned}
$$

say. Then

$$
\frac{\partial f}{\partial \sigma}=\frac{1}{2} \log \left(\frac{t}{2 \pi}\right)+O\left(\frac{1}{t^{2}}\right)
$$

which is positive for $t$ sufficiently large. On the contours, the argument of the cosine term is $n \pi+O(1 / t)$, so the cosine term is asymptotically \pm 1 uniformly in $\sigma$, giving the strict monotonicity of $\Re \Lambda(s)$.

Remark. A similar calculation to that used in Lemma 4, for $\sigma \in \mathbb{C}$ with $\Re s \neq 0$, leads to

$$
\Im \Lambda(s)=\left(\frac{t}{2 \pi}\right)^{\sigma-\frac{1}{2}}\left\{1+O\left(\frac{1}{t^{2}}\right)\right\} \sin \left(2 \vartheta_{\sigma}(t)\right) .
$$

Lemma 5. For $x, y>0$ let $P_{+}:=\frac{1}{2}+x+i y, P_{-}:=\frac{1}{2}-x+i y$. Let $\zeta(s)$ have $a$ zero of order $m$ at $s=P_{+}$for some $x$. Then $\left|\zeta^{(m)}\left(P_{-}\right)\right|>\left|\zeta^{(m)}\left(P_{+}\right)\right|$. Hence, if $m=1, \zeta^{\prime}\left(P_{+}\right) \neq \pm \zeta^{\prime}\left(P_{-}\right)$. 
Proof. Differentiating $\zeta(1-s)=\Lambda(s) \zeta(s) m$ times leads to

$$
(-1)^{m} \zeta^{(m)}\left(\overline{P_{-}}\right)=\Lambda\left(P_{+}\right) \zeta^{(m)}\left(P_{+}\right) .
$$

Because $|\Lambda(s)|=\left(\frac{t}{2 \pi}\right)^{\sigma-\frac{1}{2}}\left[1+O\left(\frac{1}{t^{2}}\right)\right]$, considering the value of the implied constant shows that $\left|\Lambda\left(P_{+}\right)\right|>1$ if $t>10$.

Lemma 6. The difference in the arguments of $\Phi(s)$ at $P_{ \pm}$satisfies the asymptotic equation

$$
\arg \Phi\left(P_{+}\right)-\arg \Phi\left(P_{-}\right)=\frac{\pi x}{2}+O\left(\frac{1}{y}\right) .
$$

Proof. First note that

$$
\begin{aligned}
\arg \Phi\left(P_{+}\right)-\arg \Phi\left(P_{-}\right)=\Im \log \Gamma & \left(\frac{1}{4}+\frac{x}{2}+\frac{i y}{2}\right) \\
& -\Im \log \Gamma\left(\frac{1}{4}-\frac{x}{2}+\frac{i y}{2}\right) .
\end{aligned}
$$

The result follows by expanding and simplifying this expression in the normal manner.

Lemma 7. The contours $\Im \Phi(s)=0$ cut the critical line at the Gram points at an angle to the horizontal, which is $-\pi /(2 \log t)$ asymptotically. They differ from intervals at that slope by $O(1 / t)$ and have equations given implicitly by

$$
\frac{t}{2} \log \frac{t}{2 \pi}-\frac{t}{2}+\frac{\pi(\sigma-1)}{4}+\frac{1}{12 t}-\frac{(\sigma-1)^{2}}{4 t}+O\left(\frac{1}{t^{3}}\right)=n \pi .
$$

Proof. Let $s=\frac{1}{2}+i t$. Then, since $\Phi(s)$ is real, $\overline{\Phi\left(\frac{1}{2}+i t\right)}=\Phi\left(\frac{1}{2}-i t\right)=\Phi\left(\frac{1}{2}+i t\right)$. Therefore

$$
\frac{\Phi\left(\frac{1}{2}-i t\right)}{\Phi\left(\frac{1}{2}+i t\right)}=1=\Lambda\left(\frac{1}{2}+i t\right)
$$

Therefore by Lemma 2, $\frac{1}{2}+i t$ is a Gram point. The remainder of the derivation is similar to that of Lemma 3 so is omitted.

\section{Averages}

Figure 3 represents averages of $\left(\Re \zeta\left(\sigma+i g_{n}\right): 1 \leq n \leq N\right)$ for values of $\sigma \in$ $\{0.0,0.1,0.3,0.5,0.6,0.8,1.0\}$. Modified values are averages of

$$
\Re \zeta\left(\sigma+i g_{n}\right)-\left(g_{n} /(2 \pi)\right)^{\frac{1}{2}}-\sigma,
$$

and by Theorem 1 they tend to 1 . Using 5120 Gram points resulted in agreement with the theorem to within 1 in $10^{4}$ for each of the displayed $\sigma$ values.

To deal analytically with these averages we need to bound cosine sums.

Lemma 8. Let $1 \leq M<N$ and let $n \geq 2$. Then

$$
\sum_{\nu=M+1}^{N} \cos \left(g_{\nu} \log n\right)=O\left(N^{\frac{1}{2}} \log N\right)
$$

where the implied constant is absolute. 

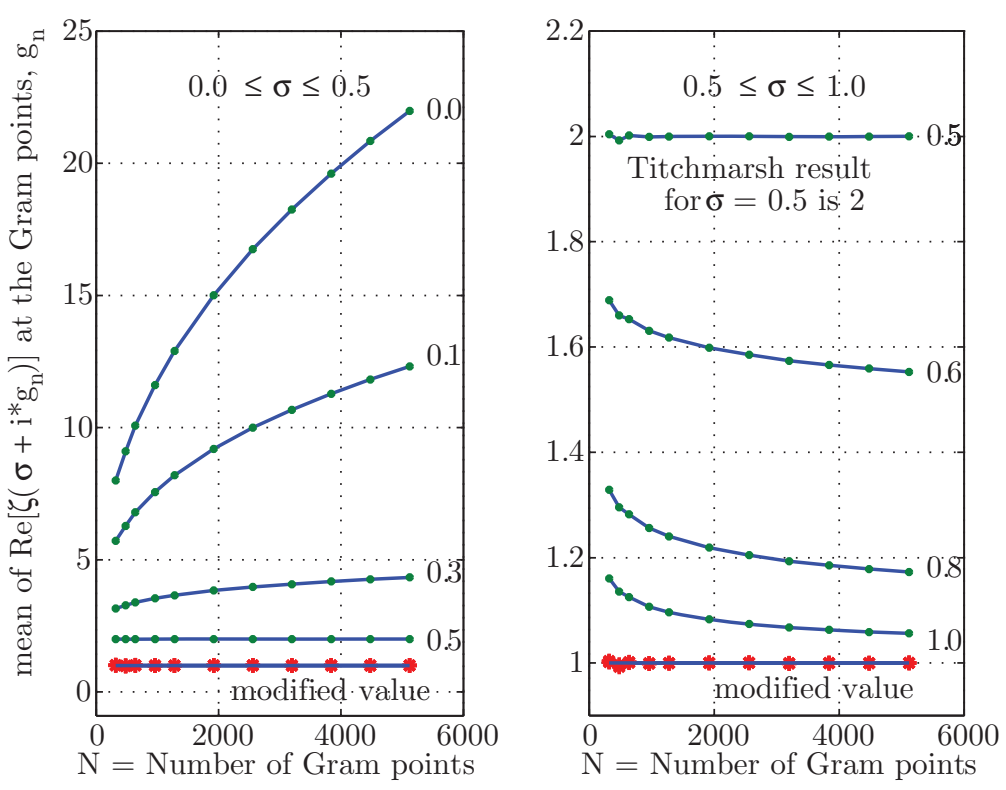

FiguRE 3. Averages for $\Re \zeta(s)$.

Proof. We begin by following the argument set out in [11, Section 10.6]. For all $\nu \in \mathbb{R}$ with $\nu>0$, let $\vartheta\left(t_{\nu}\right)=\nu \pi$, so for the integral $\nu, t_{\nu}=g_{\nu}$, and define the function $\phi$ by

Then

$$
\phi(\nu):=\frac{t_{\nu} \log n}{2 \pi}
$$

and therefore

$$
\phi^{\prime}(\nu)=\frac{\log n}{2 \vartheta^{\prime}\left(t_{\nu}\right)}
$$

$$
\phi^{\prime \prime}(\nu)=-\pi \log n \frac{\vartheta^{\prime \prime}\left(t_{\nu}\right)}{2 \vartheta^{\prime}\left(t_{\nu}\right)^{3}} .
$$

Note that, because the negative power of $t$ terms in $\vartheta(t)$ are continuous,

$$
\begin{aligned}
\vartheta(t) & =\frac{1}{2} t \log t+O(t), \vartheta^{\prime}(t)=\frac{1}{2} \log t+O(1), \\
\vartheta^{\prime \prime}(t) & =\frac{1}{2 t}+O\left(\frac{1}{t^{2}}\right), \text { and } t_{\nu} \sim \frac{2 \pi \nu}{\log \nu} .
\end{aligned}
$$

Therefore

$$
-\phi^{\prime \prime}(\nu)=\frac{2 \pi \log n}{t_{\nu} \log ^{3} t_{\nu}}+O\left(\frac{1}{t_{\nu}^{2}}\right) .
$$

Hence if $L:=2 \pi \log n / t_{N} \log ^{3} t_{N}$ and $M$ is sufficiently large, then, for all $\nu$ with $M<\nu \leq N,-\phi^{\prime \prime}(\nu) \geq L>0$.

Now we depart from [11], applying [7, Corollary 8.12] with $f=-\phi$. This gives directly

$$
\sum_{\nu=M+1}^{N} \cos \left(t_{\nu} \log n\right)=O\left(t_{N}^{\frac{1}{2}} \log t_{N}\right)=O\left(N^{\frac{1}{2}} \log N\right) .
$$


Finally note that the implied constant depends only on $1 / \log n$, so it can be made absolute, and the proof of the lemma is complete.

Now we are able to give the proof of Theorem 1 which we restate:

Let $N$ be a positive integer and let $\sigma>0$ be a given positive real number. Then as $N \rightarrow \infty$ :

$$
\begin{aligned}
\sum_{1 \leq n \leq N} & {\left[\Re \zeta\left(\sigma+i g_{n}\right)-1-\left(\frac{g_{n}}{2 \pi}\right)^{\frac{1}{2}-\sigma}\right] } \\
= & O\left(N^{\max \left\{1-\frac{\sigma}{2}, \frac{1}{2}\right\}}(\log N)^{\max \left\{\frac{1}{2}+\frac{\sigma}{2}, 2\right\}}\right)
\end{aligned}
$$

uniformly in $\sigma>0$.

Proof. (1) First let $3 / 2 \geq \sigma_{N}>1$, where $\sigma_{N}$ will be chosen later, and let $\sigma \geq \sigma_{N}$. Then, by Lemma 8 and because $\left(g_{n} / 2 \pi\right)^{\frac{1}{2}-\sigma} \ll \sqrt{\log n / n}$, we have

$$
\begin{aligned}
& \sum_{1 \leq n \leq N}\left[\Re \zeta\left(\sigma+i g_{n}\right)-1-\left(\frac{g_{n}}{2 \pi}\right)^{\frac{1}{2}-\sigma}\right] \\
= & O\left(N^{\frac{1}{2}} \log ^{\frac{1}{2}} N\right)+\sum_{k=2}^{\infty} \frac{1}{k^{\sigma}} \sum_{1 \leq n \leq N} \cos \left(g_{n} \log k\right) \ll \frac{1}{\sigma_{N}-1} N^{\frac{1}{2}} \log N,
\end{aligned}
$$

for $\sigma \geq \sigma_{N}$, where the implied constant is absolute.

(2) Now let $0<\sigma<\sigma_{N}$ and consider the approximate functional equation [11, page 79, Eqn. 4.12.4] in the form

$$
\zeta(s)=\sum_{1 \leq n \leq \sqrt{\frac{t}{2 \pi}}} \frac{1}{n^{s}}+\chi(s) \sum_{1 \leq n \leq \sqrt{\frac{t}{2 \pi}}} \frac{1}{n^{1-s}}+O\left(\frac{1}{t^{\sigma / 2}}\right)
$$

where $t>0$ and $s=\sigma+i t$. Note that $\chi(s)=1 / \Lambda(s)$, so, using Lemma 4 ,

$$
\begin{aligned}
\cos \left(2 \vartheta_{\sigma}\left(g_{\nu}\right)\right) & =1+O\left(\frac{1}{g_{\nu}^{2}}\right), \\
\sin \left(2 \vartheta_{\sigma}\left(g_{\nu}\right)\right) & =O\left(\frac{1}{g_{\nu}}\right), \\
\Re \chi\left(g_{\nu}\right) & =\left(\frac{g_{\nu}}{2 \pi}\right)^{\frac{1}{2}-\sigma}\left\{1+O\left(\frac{1}{g_{\nu}^{2}}\right)\right\} \cos \left(2 \vartheta_{\sigma}\left(g_{\nu}\right)\right), \text { and } \\
\Im \chi\left(g_{\nu}\right) & =-\left(\frac{g_{\nu}}{2 \pi}\right)^{\frac{1}{2}-\sigma}\left\{1+O\left(\frac{1}{g_{\nu}^{2}}\right)\right\} \sin \left(2 \vartheta_{\sigma}\left(g_{\nu}\right)\right) .
\end{aligned}
$$

Now take the real part of the approximate functional equation, and evaluate both sides with $t=g_{\nu}$, corresponding to an even value of $n$ in equation (1) of 
Lemma 3, to obtain

$$
\begin{aligned}
\Re \zeta(\sigma+ & \left.i g_{\nu}\right)=1+\left(\frac{g_{\nu}}{2 \pi}\right)^{\frac{1}{2}-\sigma}\left\{1+O\left(\frac{1}{g_{\nu}^{2}}\right)\right\}^{2} \\
& +\sum_{2 \leq n \leq \sqrt{\frac{g_{\nu}}{2 \pi}}} \cos \left(g_{\nu} \log n\right)\left[\frac{1}{n^{\sigma}}+\left(\frac{g_{\nu}}{2 \pi}\right)^{\frac{1}{2}-\sigma}\left\{1+O\left(\frac{1}{g_{\nu}^{2}}\right)\right\}^{2} \frac{1}{n^{1-\sigma}}\right] \\
& -\sum_{2 \leq n \leq \sqrt{\frac{g_{\nu}}{2 \pi}}} \sin \left(g_{\nu} \log n\right) \frac{1}{n^{1-\sigma}}\left(\frac{g_{\nu}}{2 \pi}\right)^{\frac{1}{2}-\sigma} O\left(\frac{1}{g_{\nu}}\right)+O\left(\frac{1}{g_{\nu}^{\sigma / 2}}\right) .
\end{aligned}
$$

Therefore

$$
\begin{aligned}
f_{0}(\nu, \sigma) & :=\Re \zeta\left(\sigma+i g_{\nu}\right)-1-\left(\frac{g_{\nu}}{2 \pi}\right)^{\frac{1}{2}-\sigma} \\
= & \sum_{2 \leq n \leq \sqrt{g_{\nu} / 2 \pi}} \frac{\cos \left(g_{\nu} \log n\right)}{n^{\sigma}}+\left(\frac{g_{\nu}}{2 \pi}\right)^{\frac{1}{2}-\sigma} \sum_{2 \leq n \leq \sqrt{g_{\nu} / 2 \pi}} \frac{\cos \left(g_{\nu} \log n\right)}{n^{1-\sigma}} \\
& +O\left(\frac{1}{g_{\nu}^{\sigma / 2}}\right)
\end{aligned}
$$

where the third term and three error terms have been absorbed into one error term.

Call the first term on the right $f_{1}(\nu, \sigma)$ and the second $f_{2}(\nu, \sigma)$, so

$$
\begin{aligned}
\sum_{M<\nu \leq N} f_{0}(\nu, \sigma) & =\sum_{M<\nu \leq N} f_{1}(\nu, \sigma)+\sum_{M<\nu \leq N} f_{2}(\nu, \sigma) \\
& +O\left(\sum_{M<\nu \leq N} \frac{1}{g_{\nu}^{\sigma / 2}}\right) \\
& =F_{1}(\sigma)+F_{2}(\sigma)+O\left(N^{1-\frac{\sigma}{2}}(\log N)^{\frac{\sigma}{2}}\right) .
\end{aligned}
$$

(3) Consider in this last equation the term $F_{1}(\sigma)$. Let

$$
\tau:=\max \left(g_{M+1}, 2 \pi n^{2}\right) .
$$

Then, by Lemma 8

$$
\begin{aligned}
F_{1}(\sigma) & =\sum_{\nu=M+1}^{N} \sum_{2 \leq n \leq \sqrt{g_{\nu} / 2 \pi}} \frac{\cos \left(g_{\nu} \log n\right)}{n^{\sigma}} \\
& =\sum_{2 \leq n \leq \sqrt{g_{N} / 2 \pi}} \frac{1}{n^{\sigma}} \sum_{\tau \leq g_{\nu} \leq g_{N}} \cos \left(g_{\nu} \log n\right) \\
& =O\left(g_{N}^{\frac{1-\sigma}{2}+\frac{1}{2}} \log g_{N}\right)=O\left(N^{1-\frac{\sigma}{2}}(\log N)^{\frac{\sigma}{2}}\right) .
\end{aligned}
$$


(4) Consider $F_{2}(\sigma)$ and define $t(x):=x / \log x$. Then, again using Lemma 8 ,

$$
\begin{aligned}
& \sum_{\tau \leq g_{\nu} \leq g_{N}} g_{\nu}^{\frac{1}{2}-\sigma} \cos \left(g_{\nu} \log n\right)=\sum_{\tau \leq g_{\nu} \leq g_{N}} g_{N}^{\frac{1}{2}-\sigma} \cos \left(g_{\nu} \log n\right) \\
& +\left(\sigma-\frac{1}{2}\right) \int_{\tau \leq t(x) \leq g_{N}} \sum_{\tau \leq g_{\nu} \leq t(x)} \cos \left(g_{\nu} \log n\right) t(x)^{\prime} t(x)^{-\frac{1}{2}-\sigma} d x \\
& \ll N^{1-\sigma}(\log N)^{\frac{1}{2}+\sigma}+\int_{2}^{N} \sqrt{x} \log x \cdot \frac{1}{\log x} \cdot\left(\frac{\log x}{x}\right)^{\frac{1}{2}+\sigma} d x \\
& \ll N^{1-\sigma}(\log N)^{\frac{1}{2}+\sigma}+1,
\end{aligned}
$$

where the implied constant is absolute since $\sigma<\frac{3}{2}$. Therefore

$$
\begin{aligned}
F_{2}(\sigma) & \ll \sum_{2 \leq n \leq \sqrt{\frac{g N}{2 \pi}}} \frac{1}{n^{1-\sigma}}\left(N^{1-\sigma}(\log N)^{\frac{1}{2}+\sigma}+1\right) \\
& \ll N^{1-\frac{\sigma}{2}}(\log N)^{\frac{\sigma}{2}+\frac{1}{2}}+\left(\frac{N}{\log N}\right)^{\frac{\sigma}{2}} .
\end{aligned}
$$

Now define a real-valued function

$$
g_{N}(x):=N^{1-\frac{x}{2}}(\log N)^{\frac{x}{2}+\frac{1}{2}}+N^{\frac{x}{2}}(\log N)^{-\frac{x}{2}} .
$$

On the domain $\left(0, \frac{3}{2}\right), g(x)$ has a unique minimum at

$$
x=\frac{\log N+\frac{1}{2} \log \log N}{\log N-\log \log N}
$$

so if we define $\sigma_{N}:=\frac{\log N}{\log (N / \log N)}$ we get, on the one hand, the upper bound in (1) above

$$
\frac{1}{\sigma_{N}-1} N^{\frac{1}{2}} \log N \leq \frac{N^{\frac{1}{2}}(\log N)^{2}}{\log \log N}
$$

On the other hand, evaluating $g\left(\sigma_{N}\right)$, and assuming $N$ is sufficiently large that $\log \log N / \log N \leq \frac{1}{2}$, we get, after a little manipulation, $g\left(\sigma_{N}\right) \leq N^{\frac{1}{2}} \log N$. So for $\sigma \in\left[1, \sigma_{N}\right]$ we have

$$
g(\sigma) \leq g(1) \leq N^{\frac{1}{2}}(\log N)^{2}
$$

and on $(0,1]$ we can use the bound $g(\sigma) \ll N^{1-\frac{\sigma}{2}}(\log N)^{\frac{\sigma}{2}+\frac{1}{2}}$. Therefore

$$
F_{2}(\sigma) \ll N^{\max \left\{1-\frac{\sigma}{2}, \frac{1}{2}\right\}}(\log N)^{\max \left\{\frac{\sigma}{2}+\frac{1}{2}, 2\right\}} .
$$

(5) Combining the estimates from (1), (2), (3) and (4) gives the final result for $0<\sigma$. Note that we can absorb the neglected first $M$ terms into the sum because the exponent of $N$ is positive in the given range. This completes the proof of Theorem 1.

Numerical evidence strongly suggests this result extends to the line $\sigma=0$ and beyond to the left. Indeed, we conjecture that this is so.

Corollary 9. Let $N$ be a positive integer. Then for all $\sigma>0$,

$$
\lim _{N \rightarrow \infty} \frac{1}{N} \sum_{1 \leq n \leq N}\left[\Re \zeta\left(\sigma+i g_{n}\right)-1-\left(\frac{g_{n}}{2 \pi}\right)^{\frac{1}{2}-\sigma}\right]=0 .
$$


Because for any $\mathbf{e}>0$ and $N$ sufficiently large, we can write

$$
\sum_{1 \leq n \leq N} \Re \zeta\left(\sigma+i g_{n}\right)=N+\sum_{1 \leq n \leq N}\left(\frac{g_{n}}{2 \pi}\right)^{\frac{1}{2}-\sigma}+O\left(N^{\max \left\{1-\frac{\sigma}{2}, \frac{1}{2}\right\}+\mathbf{e}}\right),
$$

we get:

Corollary 10. For all $\sigma$ with $0<\sigma$ and $\mathbf{e}$ with $0<\mathbf{e}<1$ the inequality

$$
\sum_{1 \leq n \leq N} \Re \zeta\left(\sigma+i g_{n}\right) \geq(1-\mathbf{e}) N
$$

holds for all $N$ sufficiently large.

Corollary 11. For each $\sigma$ with $0<\sigma<1$ there exist an infinite number of positive integers $n$ with $\Re \zeta\left(\sigma+i g_{n}\right)>0$.

\section{ACKNOWLEDGMENT}

We acknowledge the support of the University of Waikato. Numerical calculations and plots were made using Mathematica and MATLAB. The many helpful comments of a referee are also gratefully acknowledged.

\section{REFERENCES}

[1] R. Backlund, Sur les zéros de la fonction $\zeta(s)$ de Riemann, C. R. Acad. Sci. Paris, 158 (1914), 1979-1982.

[2] R. P. Brent, J. van de Lune, H. J. J. Riele and D. T. Winter, On the zeros of the Riemann zeta function in the critical strip. II, Math. Comp. 39 (1982), 681-688. MR669660 (83m:10067)

[3] K. A. Broughan, Holomorphic flows on simply connected domains have no limit cycle, Meccanica 38(6) (2003), 699-709. MR2028269 (2004m:37092)

[4] H. M. Edwards, Riemann's zeta function, Dover, 2001. MR.1854455 (2002g:11129)

[5] J. -P. Gram, Sur les zéros de la fonction $\zeta(s)$ de Riemann, Acta Math. 27, (1903), 289-304. MR 1554986

[6] J. I. Hutchinson, On the roots of the Riemann zeta-function, Trans. Amer. Math. Soc. 27 (1925), 49-60. MR.1501297

[7] H. Iwaniec and E. Kowalski, Analytic Number Theory, American Mathematical Society, 2004. MR2061214(2005h:11005)

[8] D. H. Lehmer, On the roots of the Riemann zeta-function, Acta Math. 95 (1956), 291-298. MR0086082 (19:121a)

[9] J. B. Rosser, J. M. Yohe and L. Schoenfeld, Rigorous computation and the zeros of the Riemann zeta function, Proceedings of the IFIP Congress, vol 1: Mathematics, 70-76, Amsterdam, 1968, North-Holland. MR0258245 (41:2892)

[10] E. C. Titchmarsh, On van der Corput's method and the zeta-function of Riemann, (IV). Quart. J. Math. Oxford Ser. 5, (1934), 98-105.

[11] E. C. Titchmarsh, revised by D. R. Heath-Brown, The theory of the Riemann zeta-function, Second edition, Oxford, 1994. MR882550 (88c:11049)

[12] T. Trudgian, On the success and failure of Gram's Law and the Rosser Rule, Acta Arith. 148, (2011), 225-256. MR.2794929

University of Waikato, Hamilton, New Zealand

E-mail address: kab@waikato.ac.nz

University of Waikato, Hamilton, New Zealand

E-mail address: arbus@math.waikato.ac.nz 